\title{
ERRATUM
}

doi:10.1038/nature08202

\section{Slow earthquakes triggered by typhoons}

ChiChing Liu, Alan T. Linde \& I. Selwyn Sacks

Nature 459, 833-836 (2009)

In this Letter, address 1 was incorrectly listed. This oversight has now been rectified. 